\title{
Delay-Dependent and Order-Dependent Guaranteed Cost Control for Uncertain Fractional-Order Delayed Linear Systems
}

\author{
Fei $\mathrm{Qi}^{1}{ }^{1}$, Yi Chai ${ }^{1, *}$, Liping Chen ${ }^{2}$ and José A. Tenreiro Machado ${ }^{3}$ \\ 1 School of Automation, Chongqing University, Chongqing 400044, China; fei_qi@cqu.edu.cn \\ 2 School of Electrical Engineering and Automation, Hefei University of Technology, Hefei 230009, China; \\ lip_chenhut@hfut.edu.cn \\ 3 Department of Electrical Engineering, Institute of Engineering, Polytechnic of Porto, R. Dr. António \\ Bernardino de Almeida, 431, 4249-015 Porto, Portugal; jtm@isep.ipp.pt \\ * Correspondence: chaiyi@cqu.edu.cn
}

check for

updates

Citation: Qi, F.; Chai, Y.; Chen, L.;

Tenreiro Machado, J.A.

Delay-Dependent and

Order-Dependent Guaranteed Cost Control for Uncertain

Fractional-Order Delayed Linear

Systems. Mathematics 2021, 9, 41.

https://dx.doi.org/10.3390/

math9010041

Received: 12 November 2020

Accepted: 23 December 2020

Published: 27 December 2020

Publisher's Note: MDPI stays neutral with regard to jurisdictional claims in published maps and institutional affiliations.

Copyright: () 2020 by the authors. Licensee MDPI, Basel, Switzerland. This article is an open access article distributed under the terms and conditions of the Creative Commons Attribution (CC BY) license (https: / / creativecommons.org/ licenses/by/4.0/).

\begin{abstract}
This paper addresses the guaranteed cost control problem of a class of uncertain fractionalorder (FO) delayed linear systems with norm-bounded time-varying parametric uncertainty. The study is focused on the design of state feedback controllers with delay such that the resulting closed-loop system is asymptotically stable and an adequate level of performance is also guaranteed. Stemming from the linear matrix inequality (LMI) approach and the FO Razumikhin theorem, a delay- and order-dependent design method is proposed with guaranteed closed-loop stability and cost for admissible uncertainties. Examples illustrate the effectiveness of the proposed method.
\end{abstract}

Keywords: fractional-order systems; guaranteed cost control; input delay; LMI approach

\section{Introduction}

In recent years, fractional calculus and fractional-order $(\mathrm{FO})$ systems became an important topic of research in science and engineering [1,2]. Integer-order modeling techniques may not lead to the best descriptions of a system dynamics, especially concerning the systems with heredity and memory properties. Successful applications in control, mathematics, mechanics, biology, chemistry, and signal and image processing [3-10] have shown that fractional calculus offers a new and deeper perspective in system modeling that overcomes the shortcomings of classical differential constitutive models [11,12].

Over the past ten years, stability, as well as robust control for FO uncertain linear systems have been key research topics in the field of control theory. A method of observerbased control for FO uncertain linear systems with the fractional commensurate order $1<\alpha<2$ based on linear matrix inequality (LMI) approach was discussed in [13]. The stability and stabilization analysis of FO linear time-invariant systems with different derivative orders were considered in [14]. The asymptotic stability and stabilization of FO linear systems with time-varying structured uncertainties and time-varying delay were addressed in [15]. The robust stability and stabilization of a class of fractional order systems under input saturation using the Lyapunov method was studied in [16]. A necessary and sufficient condition for testing the robust D-stability of linear time-invariant (LTI) general FO control systems was derived in [17]. Other studies regarding these topics can be found in [18-20] and references therein.

The stability is a primary requirement for designing a controller for a real plant. Moreover it is also desirable but difficult to design a control system which not only preserves stability, but can also guarantees an adequate level of performance. To cope with the problem, Chang and Peng proposed an alternative way, namely the so-called guaranteed cost control approach [21]. The main advantage ia that it guarantees the robust stability of the closed-loop system, and ensures that the deteriorated performance caused by the system uncertainty is still inferior to the upper bound of the previously prescribed performance 
index. This approach has provided a certain degree of understanding of the extent of system performance degradation [22]. Some significant results on guaranteed cost control for integer-order systems have been put forward by several researchers [22-31]. Nonetheless, it should be pointed out that input delays are unavoidable due to the time taken for transportation of materials and transmission of signals in many physical phenomena, such as in biological systems and chemical processes. The existence of input delay will eventually lead to instability and poor system performance. Therefore, the stabilisation of input-delayed systems is one fundamental problem. However, to our best knowledge, we find limited results about the guaranteed cost control of FO uncertain delayed linear systems by using feedback controller with input delay. Motivated by this observation, the paper focusses on the guaranteed cost control controller design problem of uncertain FO linear systems with time-delay. The time delays can appear both in the state and input. The problem consists of the design of state-feedback controllers such that the resulting closed-loop system is robustly stable and a specified integral-quadratic cost function has an upper bound for all delays within the given intervals. Delay-dependent and order-dependent conditions for the solvability of this problem are obtained in terms of matrix inequalities.

The rest of this paper is organized as follows. Preliminary notions and problem description will be discussed in Section 2. The solution to this problem is given in Section 3. Two examples illustrating performance of the proposed strategy are presented in Section 4 . Finally, the conclusions are given in Section 5.

\section{Preliminary nOtions and Problem Description}

The following symbols are used in the follow-up stands for: $C\left([a, b], R^{n}\right)$ the set of continuous functions mapping the interval $[a, b]$ to $R^{n}, I$ is the identity matrix with the appropriate dimension, and the symbol $\star$ denotes the elements below the main diagonal of a symmetric block matrix. The superscript $T$ represents the transpose, $\operatorname{diag}\{\cdot\}$ stands for the diagonal matrix, and $X>0(<0)$ is a symmetric positive definite (negative definite) matrix. The symbol $C=C\left([-\tau, 0], R^{n}\right), x_{t}(\theta) \in C$ is a segment of function defined as $x_{t}(\theta)=x(t+\theta),-\tau \leq \theta \leq 0$.

The Riemann-Liouville (RL), Grünwald-Letnikov (GL) and Caputo (C) formulations are commonly used definitions of fractional integrals and derivatives. Here the Caputo definition is used due to its convenience in engineering applications.

Definition 1. [32] The fractional integral of order $\alpha>0$ of a function $x(t)$ is:

$$
I_{t_{0}, t}^{\alpha} x(t)=\frac{1}{\Gamma(\alpha)} \int_{t_{0}}^{t}(t-\tau)^{\alpha-1} x(\tau) d \tau
$$

where $\Gamma(\cdot)$ is the Gamma function, $\Gamma(s)=\int_{0}^{\infty} t^{s-1} e^{-t} d t$.

Definition 2. [32] The Caputo derivative of order $\alpha$ of a function $x(t)$ is:

$$
{ }^{C} D_{t_{0}, t}^{\alpha} x(t)=I_{t_{0}, t}^{n-\alpha} \frac{d^{n}}{d r^{n}} x(t)=\frac{1}{\Gamma(n-\alpha)} \int_{t_{0}}^{t}(t-\tau)^{n-a-1} x^{(n)}(\tau) d \tau,
$$

where $n-1<\alpha<n \in \mathbb{Z}^{+}$.

In the following, the notation $D^{\alpha}$ is chosen as the Caputo derivative ${ }^{C} D_{t_{0}, t}^{\alpha}$. Consider the following $n$-dimensional FO linear uncertain delayed system

$$
\left\{\begin{aligned}
D^{\alpha} x(t) & =(A+\Delta A) x(t)+\left(A_{\tau}+\Delta A_{\tau}\right) x(t-\tau)+(B+\Delta B) u(t), t>0, \\
x(t) & =\phi(t), \quad t \in[-\tau, 0], \\
y(t) & =C x(t)
\end{aligned}\right.
$$


where $n-1<\alpha<n \in Z^{+}$, and $\Gamma(\cdot)$ is the Gamma function, $\Gamma(s)=\int_{0}^{\infty} t^{s-1} e^{-t} d t$. Moreover, $x(t)=\left(x_{1}(t), \cdots, x_{n}(t)\right)^{T} \in R^{n}$ represents system the state vector, the FO $\alpha$ belongs to the interval $(0,1]$. The symbols $A \in R^{n \times n}$ and $A_{\tau} \in R^{n \times n}$ represent the nominal and the state delayed matrices, $\tau$ denotes the time delay and $x(t)=\phi(t)$ is a continuous vector-valued initial condition on $[-\tau, 0]$. The uncertain matrices $\Delta A, \Delta A_{\tau}$ and $\Delta B$ are time-varying with appropriate dimensions subject to the following form:

$$
\left[\begin{array}{ccc}
\Delta A & \Delta A_{\tau} & \Delta B
\end{array}\right]=E_{1} H(t)\left[\begin{array}{lll}
F_{1} & F_{2} & F_{3}
\end{array}\right],
$$

where $E_{1}, F_{1}, F_{2}$ and $F_{3}$ are known constant real matrices with appropriate dimensions, and $H(t)$ is the unknown time-varying matrix satisfying

$$
H^{T}(t) H(t) \leq I
$$

Given positive definite symmetric matrices $Q_{1}$ and $Q_{2}$, the following the cost function for system (1) is used

$$
J=\frac{1}{\Gamma(\alpha)} \int_{t_{0}}^{T_{1}}\left(T_{1}-s\right)^{\alpha-1}\left(x^{\mathrm{T}}(s) Q_{1} x(s)+u^{\mathrm{T}}(s) Q_{2} u(s)\right) d s,
$$

where $T_{1}>0$, and $T_{1}>\tau$

Associated with the cost, the cost guaranteed controller is defined as follows:

Definition 3. For the FO dynamic system (1) and the cost function (2), if there is a control law $u$ and a positive constant $J^{*}$, so that for all the admissible uncertainties and time-delays, the obtained closed-loop system is asymptotically stable and the closed-loop value of the cost function satisfies $J<J^{*}$, then $J^{*}$ is called cost guaranteed.

The uncertain FO system (1) is said to be the cost guaranteed control using linear state-feedback controller with delay if there exists a state-feedback controller

$$
\left\{\begin{array}{l}
u(t)=-K x(t-\tau), \quad t \geq \tau, \\
u(t)=0, \quad 0<t<\tau,
\end{array}\right.
$$

such that the following closed-loop system is asymptotically stable and an adequate level of performance is also guaranteed

$$
\left\{\begin{aligned}
D^{\alpha} x(t) & =(A+\Delta A) x(t)+\left(A_{\tau}+\Delta A_{\tau}-B K-\Delta B K\right) x(t-\tau), \\
x(t) & =\phi(t), \quad t \in[-\tau, 0] .
\end{aligned}\right.
$$

Remark 1. In practical engineering applications, the controller starts working when time is greater than 0 . That is, there is not control input in the delayed system when $t \in[0, \tau]$, the system is an open loop one.

For this end, the following Lemmas are presented firstly.

Lemma 1. [32] If $x(t) \in C^{n}([0,+\infty), R)$ and $n-1<\alpha<n$, then the following formula holds

$$
I_{t_{0}, t}^{\alpha} D^{\alpha} x(t)=x(t)-\sum_{k=0}^{n-1} \frac{t^{k}}{k !} x^{k}(0),
$$

where $I_{t_{0}, t}^{\alpha} x(t)=\frac{1}{\Gamma(\alpha)} \int_{t_{0}}^{t}(t-\tau)^{\alpha-1} x(\tau) d \tau$. 
Lemma 2. [33] Let $x(t) \in R^{n}$ be a differentiable vector-value function. Then, for any time instant $t \geq t_{0}$

$$
D^{\alpha}\left(x^{\mathrm{T}}(t) P x(t)\right) \leq\left(x^{\mathrm{T}}(t) P\right) D^{\alpha} x(t)+\left(D^{\alpha} x(t)\right)^{\mathrm{T}} P x(t)
$$

where $P \in R^{n \times n}$ is a symmetric positive definite matrix, $\alpha \in(0,1)$.

Lemma 3. [34] In the nonlinear delayed system

$$
D^{\alpha} x(t)=f\left(t, x_{t}\right)
$$

the initial states of Caputo system (5) is defined as $x_{t_{0}}=\varphi(t)$, which a continuous vector-valued function on $[-\tau, 0]$. The function $f:\left[t_{0}, \infty\right] \times C_{\tau} \rightarrow R^{n}$ is piecewise continuous in $t$ and locally Lipschitz in $x$ on $\left[t_{0}, \infty\right]$.

Suppose that $\omega_{1}, \omega_{2}: \mathbb{R} \rightarrow \mathbb{R}$ are continuous nondecreasing functions, $\omega_{1}(s)$ and $\omega_{2}(s)$ are positive for $s>0$, and $\omega_{1}(0)=\omega_{2}(0)=0$, and $\omega_{2}$ strictly increasing. If there exists $a$ continuously differentiable function $V: \mathbb{R} \times \mathbb{R}^{n} \rightarrow R$ such that

$$
\omega_{1}(\|x\|) \leq V(t, x) \leq \omega_{2}(\|x\|), \text { for } t \in \mathbb{R}, x \in \mathbb{R}^{n},
$$

and for any given $\mathrm{t}_{0} \in \mathbb{R}$ the Caputo fractional derivative of $V$ along the solution $x(t)$ of the FO system (5) satisfies

$$
\left\{\begin{array}{l}
D^{\alpha} V(t, x(t)) \leq 0 \\
\text { whenever } \sup _{t_{0}-\tau \leqslant \theta \leq t} V(\theta, x(\theta))=V(t, x(t)), \quad t \geqslant t_{0}
\end{array}\right.
$$

then the FO system (5) is uniformly stable.

If, in addition, there exist two constants $p, q>0$ with $p<q$ such that

$$
D^{\alpha} V(t, x(t)) \leq-q V(t, x(t))+p \sup _{-\tau \leqslant \theta \leqslant 0} V(t+\theta, x(t+\theta)),
$$

for $t \geqslant t_{0}$, then the FO system (5) is globally uniformly asymptotically stable.

Lemma 4. [35] Given matrices $Q=Q^{T}, H, E$ and $R=R^{T}>0$ of appropriate dimensions, the relationship

$$
Q+H F E+E^{T} F^{T} H^{T}<0,
$$

for all $F$ satisfying $F^{T} F \leq R$, if and only if there exists some $\lambda>0$ such that

$$
Q+\lambda H H^{T}+\lambda^{-1} E^{T} R E<0 .
$$

Lemma 5. [36] (Schur Complement) For matrices $M$, so that $M_{11}^{-1}$ exists, the following inequality forms are equivalent:

$$
\begin{aligned}
M & =\left(\begin{array}{cc}
M_{11} & M_{12} \\
* & M_{22}
\end{array}\right)<0, \\
M_{11} & <0, \quad M_{22}-M_{12}^{T} M_{11}^{-1} M_{12}<0 \\
M_{22} & <0, \quad M_{11}-M_{12}^{T} M_{22}^{-1} M_{12}<0 .
\end{aligned}
$$




\section{Main Results}

An LMI sufficient condition for delayed state feedback guaranteed cost control laws for the FO uncertain system (1) will be given in the subsection.

Theorem 1. For two given symmetric positive-definite matrix $Q_{1}$ and $Q_{2}$, the asymptotic stability of the following closed-loop system (4) is assured and the guaranteed cost control performance is achieved by the control law $u(t)$, if there exist a symmetric positive-definite matrix $\bar{P}$, any appropriately dimensioned matrix $Y$ and a positive scalar $\lambda$ such that the following LMI holds

$$
\left[\begin{array}{cccccccc}
\Gamma_{11} & \Gamma_{12} & \Gamma_{13} & \bar{P} F_{1}^{\mathrm{T}} & \lambda E & \bar{P} Q_{1} & 0 & \tau^{\alpha} \alpha^{-1} \bar{P} \\
\star & -\bar{P} & \Gamma_{23} & \Gamma_{24} & 0 & 0 & Y^{\mathrm{T}} Q_{2} & 0 \\
\star & \star & -\tau^{\alpha} \alpha^{-1} I & 0 & \lambda \tau^{\alpha} \alpha^{-1} E & 0 & 0 & 0 \\
\star & \star & \star & -\lambda I & 0 & 0 & 0 & 0 \\
\star & \star & \star & \star & -\lambda I & 0 & 0 & 0 \\
\star & \star & \star & \star & \star & -Q_{1} & 0 & 0 \\
\star & \star & \star & \star & \star & \star & -Q_{2} & 0 \\
\star & \star & \star & \star & \star & \star & \star & -\tau^{\alpha} \alpha^{-1} I
\end{array}\right]<0
$$

where

$$
\begin{array}{lll}
\Gamma_{11}=A \bar{P}+\bar{P} A^{\mathrm{T}}+\mu \bar{P}, & \Gamma_{12}=A_{\tau} \bar{P}-B Y, & \Gamma_{13}=\tau^{\alpha} \alpha^{-1} \bar{P} A^{\mathrm{T}}, \\
\Gamma_{23}=\tau^{\alpha} \alpha^{-1}\left(\bar{P} A_{\tau}^{\mathrm{T}}-Y^{\mathrm{T}} B^{\mathrm{T}}\right), & \Gamma_{24}=\bar{P} F_{2}^{\mathrm{T}}-Y^{\mathrm{T}} F_{3}^{\mathrm{T}}, &
\end{array}
$$

and $u(t)$ is given by

$$
u(t)=-K x(t-\tau)=-Y \bar{P}^{-1} x(t-\tau)
$$

with the cost

$$
J^{*}=\lambda_{\max }\left(\bar{P}^{-1}\right)\|\phi\|^{2} .
$$

Proof. Constructing the Lyapunov function candidate $V(x(t))=x^{\mathrm{T}}(t) P x(t)$ and calculating the derivative of $V(x(t))$ along system (1), according to Lemma 2, yields

$$
\begin{aligned}
D^{\alpha} V(x(t)) & +x^{\mathrm{T}}(t) Q_{1} x(t)+x^{\mathrm{T}}(t-\tau) K^{\mathrm{T}} Q_{2} K x(t-\tau) \\
& \leq\left(x^{\mathrm{T}}(t) P\right) D^{\alpha} x(t)+\left(D^{\alpha} x(t)\right)^{\mathrm{T}} P x(t)+x^{\mathrm{T}}(t) Q_{1} x(t) \\
& +x^{\mathrm{T}}(t-\tau) K^{\mathrm{T}} Q_{2} K x(t-\tau) \\
& =x^{\mathrm{T}}(t) P\left((A+\Delta A) x(t)+\left(A_{\tau}+\Delta A_{\tau}-B K-\Delta B K\right) x(t-\tau)\right) \\
& +\left(x^{\mathrm{T}}(t)\left(A^{\mathrm{T}}+\Delta A^{\mathrm{T}}\right)+x^{\mathrm{T}}(t-\tau)\left(A_{\tau}^{\mathrm{T}}+\Delta A_{\tau}^{\mathrm{T}}-K^{\mathrm{T}} B^{\mathrm{T}}\right.\right. \\
& \left.\left.-K^{\mathrm{T}} \Delta B^{\mathrm{T}}\right)\right) P x(t)+x^{\mathrm{T}}(t) Q_{1} x(t)+x^{\mathrm{T}}(t-\tau) K^{\mathrm{T}} Q_{2} K x(t-\tau) \\
& =x^{\mathrm{T}}(t)\left(P A+P \Delta A+A^{\mathrm{T}} P+\Delta A^{\mathrm{T}} P+Q_{1}\right) x(t) \\
& +x^{\mathrm{T}}(t)\left(P A_{\tau}+P \Delta A_{\tau}-P B K-P \Delta B K\right) x(t-\tau) \\
& +x^{\mathrm{T}}(t-\tau)\left(A_{\tau}^{\mathrm{T}} P+\Delta A_{\tau}^{\mathrm{T}} P-K^{\mathrm{T}} B^{\mathrm{T}} P-K^{\mathrm{T}} \Delta B^{\mathrm{T}} P\right) x(t) \\
& +x^{\mathrm{T}}(t-\tau) K^{\mathrm{T}} Q_{2} K x(t-\tau) .
\end{aligned}
$$

For any real matrices $X=X^{\mathrm{T}}, Y$ and $Z=Z^{\mathrm{T}}$, satisfying

$$
\Theta=\left[\begin{array}{cc}
X & Y \\
Y^{\mathrm{T}} & Z
\end{array}\right] \geq 0,
$$


we have

$$
\tau^{\alpha} \alpha^{-1} \xi^{\mathrm{T}}(t) \Theta \xi(t)-\int_{t-\tau}^{t}(t-s)^{(\alpha-1)} \xi^{\mathrm{T}}(t) \Theta \xi(t) d s \geq 0,
$$

where $\xi(t)=\left[x^{\mathrm{T}}(t),\left(D^{\alpha} x(t)\right)^{\mathrm{T}}\right]^{\mathrm{T}}$.

For the sake of simplicity, let $X=Z=I$ and $Y=0$ in (8). It follows from (7) and (8) that

$$
\begin{aligned}
D^{\alpha} V(x(t)) & +x^{\mathrm{T}}(t) Q_{1} x(t)+x^{\mathrm{T}}(t-\tau) K^{\mathrm{T}} Q_{2} K x(t-\tau) \\
& \leq\left(x^{\mathrm{T}}(t) P\right) D^{\alpha} x(t)+\left(D^{\alpha} x(t)\right)^{\mathrm{T}} P x(t)+\tau^{\alpha} \alpha^{-1} \xi^{\mathrm{T}}(t) \Theta \xi(t) \\
& -\int_{t-\tau}^{t}(t-s)^{(\alpha-1)} \xi^{\mathrm{T}}(t) \Theta \xi(t) d s \\
& =x^{\mathrm{T}}(t)\left(P A+P \Delta A+A^{\mathrm{T}} P+\Delta A^{\mathrm{T}} P+Q_{1}+\tau^{\alpha} \alpha^{-1} I\right. \\
& \left.+\tau^{\alpha} \alpha^{-1}\left(A^{\mathrm{T}}+\Delta A^{\mathrm{T}}\right)(A+\Delta A)\right) x(t) \\
& +x^{\mathrm{T}}(t)\left(P A_{\tau}+P \Delta A_{\tau}-P B K-P \Delta B K\right. \\
& \left.+\tau^{\alpha} \alpha^{-1}\left(A^{\mathrm{T}}+\Delta A^{\mathrm{T}}\right)\left(A_{\tau}+\Delta A_{\tau}-B K-\Delta B K\right)\right) x(t-\tau) \\
& +x^{\mathrm{T}}(t-\tau)\left(A_{\tau}^{\mathrm{T}} P+\Delta A_{\tau}^{\mathrm{T}} P-K^{\mathrm{T}} B^{\mathrm{T}} P-K^{\mathrm{T}} \Delta B^{\mathrm{T}} P\right. \\
& \left.+\tau^{\alpha} \alpha^{-1}\left(A_{\tau}^{\mathrm{T}}+\Delta A_{\tau}^{\mathrm{T}}-K^{\mathrm{T}} B^{\mathrm{T}}-K^{\mathrm{T}} \Delta B^{\mathrm{T}}\right)(A+\Delta A)\right) x(t) \\
& +x^{\mathrm{T}}(t-\tau)\left(K^{\mathrm{T}} Q_{2} K+\tau^{\alpha} \alpha^{-1}\left(A_{\tau}^{\mathrm{T}}+\Delta A_{\tau}^{\mathrm{T}}-K^{\mathrm{T}} B^{\mathrm{T}}\right.\right. \\
& \left.\left.-K^{\mathrm{T}} \Delta B^{\mathrm{T}}\right)\left(A_{\tau}+\Delta A_{\tau}-B K-\Delta B K\right)\right) x(t-\tau) \\
& -\int_{t-\tau}^{t}(t-s)^{(\alpha-1)} \xi^{\mathrm{T}}(t) \Theta \xi(t) d s,
\end{aligned}
$$

when $\theta$ is in the interval $-\tau \leq \theta \leq 0$, and $x(t)$ satisfies

$$
V(x(t+\theta))<\mu V(x(t)),
$$

for some $\mu>1$, one can obtain

$$
\mu x^{\mathrm{T}}(t) P x(t)-x^{\mathrm{T}}(t-\tau) P x(t-\tau) \geq 0 .
$$

Combining (9) and (10) results in

$$
\begin{aligned}
D^{\alpha} V(x(t)) & +x^{\mathrm{T}}(t) Q_{1} x(t)+x^{\mathrm{T}}(t-\tau) K^{\mathrm{T}} Q_{2} K x(t-\tau) \\
& \leq x^{\mathrm{T}}(t)\left(P A+P \Delta A+A^{\mathrm{T}} P+\Delta A^{\mathrm{T}} P+Q_{1}\right. \\
& +\tau^{\alpha} \alpha^{-1} I+\mu P+\tau^{\alpha} \alpha^{-1}\left(A^{\mathrm{T}}+\Delta A^{\mathrm{T}}\right)(A \\
& +\Delta A)) x(t)+x^{\mathrm{T}}(t)\left(P A_{\tau}+P \Delta A_{\tau}-P B K-P \Delta B K\right. \\
& \left.+\tau^{\alpha} \alpha^{-1}\left(A^{\mathrm{T}}+\Delta A^{\mathrm{T}}\right)\left(A_{\tau}+\Delta A_{\tau}-B K-\Delta B K\right)\right) x(t-\tau) \\
& +x^{\mathrm{T}}(t-\tau)\left(A_{\tau}^{\mathrm{T}} P+\Delta A_{\tau}^{\mathrm{T}} P-K^{\mathrm{T}} B^{\mathrm{T}} P-K^{\mathrm{T}} \Delta B^{\mathrm{T}} P\right. \\
& \left.+\tau^{\alpha} \alpha^{-1}\left(A_{\tau}^{\mathrm{T}}+\Delta A_{\tau}^{\mathrm{T}}-K^{\mathrm{T}} B^{\mathrm{T}}-K^{\mathrm{T}} \Delta B^{\mathrm{T}}\right)(A+\Delta A)\right) x(t) \\
& +x^{\mathrm{T}}(t-\tau)\left(K^{\mathrm{T}} Q_{2} K-P+\tau^{\alpha} \alpha^{-1}\left(A_{\tau}^{\mathrm{T}}+\Delta A_{\tau}^{\mathrm{T}}\right.\right. \\
& \left.\left.-K^{\mathrm{T}} B^{\mathrm{T}}-K^{\mathrm{T}} \Delta B^{\mathrm{T}}\right)\left(A_{\tau}+\Delta A_{\tau}-B K-\Delta B K\right)\right) x(t-\tau) \\
& -\int_{t-\tau}^{t}(t-s)^{(\alpha-1)} \xi^{\mathrm{T}}(t) \Theta \xi(t) d s \\
& =\eta^{\mathrm{T}}(t) \Pi \eta(t)-\int_{t-\tau}^{t}(t-s)^{(\alpha-1)} \xi^{\mathrm{T}}(t) \Theta \xi(t) d s,
\end{aligned}
$$

where $\eta(t)=\left[x^{\mathrm{T}}(t), x^{\mathrm{T}}(t-\tau)\right]^{\mathrm{T}}$,

$$
\Pi=\left[\begin{array}{cc}
\Pi_{11} & \Pi_{12} \\
\star & \Pi_{22}
\end{array}\right]
$$




$$
\begin{aligned}
\Pi_{11} & =P A+P \Delta A+A^{\mathrm{T}} P+\Delta A^{\mathrm{T}} P+Q_{1}+\tau^{\alpha} \alpha^{-1} I \\
& +\mu P+\tau^{\alpha} \alpha^{-1}\left(A^{\mathrm{T}}+\Delta A^{\mathrm{T}}\right)(A+\Delta A), \\
\Pi_{12} & =P A_{\tau}+P \Delta A_{\tau}-P B K-P \Delta B K \\
& +\tau^{\alpha} \alpha^{-1}\left(A^{\mathrm{T}}+\Delta A^{\mathrm{T}}\right)\left(A_{\tau}+\Delta A_{\tau}-B K-\Delta B K\right), \\
\Pi_{22} & =K^{\mathrm{T}} Q_{2} K-P+\tau^{\alpha} \alpha^{-1}\left(A_{\tau}^{\mathrm{T}}+\Delta A_{\tau}^{\mathrm{T}}\right. \\
& \left.-K^{\mathrm{T}} B^{\mathrm{T}}-K^{\mathrm{T}} \Delta B^{\mathrm{T}}\right)\left(A_{\tau}+\Delta A_{\tau}-B K-\Delta B K\right) .
\end{aligned}
$$

In fact, $\Pi<0$ can be rewritten as follow

$$
\Xi=\left[\begin{array}{ccc}
\Xi_{11} & \Xi_{12} & \tau^{\alpha} \alpha^{-1}\left(A^{\mathrm{T}}+\Delta A^{\mathrm{T}}\right) \\
\star & K^{\mathrm{T}} Q_{2} K-P & \Xi_{23} \\
\star & \star & -\tau^{\alpha} \alpha^{-1} I
\end{array}\right]<0,
$$

where

$$
\begin{aligned}
& \Xi_{11}=P A+P \Delta A+A^{\mathrm{T}} P+\Delta A^{\mathrm{T}} P+Q_{1}+\tau^{\alpha} \alpha^{-1} I+\mu P, \\
& \Xi_{12}=P A_{\tau}+P \Delta A_{\tau}-P B K-P \Delta B K \\
& \Xi_{23}=\tau^{\alpha} \alpha^{-1}\left(A_{\tau}^{\mathrm{T}}+\Delta A_{\tau}^{\mathrm{T}}-K^{\mathrm{T}} B^{T}-K^{\mathrm{T}} \Delta B^{\mathrm{T}}\right) .
\end{aligned}
$$

It is straightforward to see that (12) can be written in the form:

$$
\begin{aligned}
& {\left[\begin{array}{ccc}
\Sigma_{11} & \Sigma_{12} & \tau^{\alpha} \alpha^{-1} A^{\mathrm{T}} \\
\star & K^{\mathrm{T}} Q_{2} K-P & \tau^{\alpha} \alpha^{-1}\left(A_{\tau}^{\mathrm{T}}-K^{\mathrm{T}} B^{\mathrm{T}}\right) \\
\star & \star & -\tau^{\alpha} \alpha^{-1} I
\end{array}\right] } \\
+ & {\left[\begin{array}{c}
P E \\
0 \\
\tau^{\alpha} \alpha^{-1} E
\end{array}\right] H(t)\left[\begin{array}{ccc}
F_{1} & F_{2}-F_{3} K & 0
\end{array}\right] } \\
+ & {\left[\begin{array}{c}
F_{1}^{\mathrm{T}} \\
F_{2}^{\mathrm{T}}-K^{\mathrm{T}} F_{3}^{\mathrm{T}} \\
0
\end{array}\right] H^{\mathrm{T}}(t)\left[\begin{array}{ccc}
E^{\mathrm{T}} P & 0 & \tau^{\alpha} \alpha^{-1} E^{T}
\end{array}\right]<0 . }
\end{aligned}
$$

From Lemma 4, there exist scalars $\lambda$ such that

$$
\begin{aligned}
& {\left[\begin{array}{ccc}
\Sigma_{11} & \Sigma_{12} & \tau^{\alpha} \alpha^{-1} A^{\mathrm{T}} \\
\star & K^{\mathrm{T}} Q_{2} K-P & \tau^{\alpha} \alpha^{-1}\left(A_{\tau}^{\mathrm{T}}-K^{\mathrm{T}} B^{\mathrm{T}}\right) \\
\star & \star & -\tau^{\alpha} \alpha^{-1} I
\end{array}\right] } \\
+ & \lambda\left[\begin{array}{c}
P E \\
0 \\
\tau^{\alpha} \alpha^{-1} E
\end{array}\right]\left[\begin{array}{lll}
E^{\mathrm{T}} P & 0 & \tau^{\alpha} \alpha^{-1} E^{\mathrm{T}}
\end{array}\right] \\
+ & \lambda^{-1}\left[\begin{array}{c}
F_{1}^{\mathrm{T}} \\
F_{2}^{\mathrm{T}}-K^{\mathrm{T}} F_{3}^{\mathrm{T}} \\
0
\end{array}\right]\left[\begin{array}{lll}
F_{1} & F_{2}-F_{3} K & 0
\end{array}\right]<0,
\end{aligned}
$$

where

$$
\begin{aligned}
& \Sigma_{11}=P A+A^{\mathrm{T}} P+Q_{1}+\tau^{\alpha} \alpha^{-1} I+\mu P, \\
& \Sigma_{12}=P A_{\tau}-P B K .
\end{aligned}
$$


Substituting (13) in to (12), and using Lemma 5, we obtain that $\Pi<0$ is equivalent to

$$
\Delta=\left[\begin{array}{ccccc}
\Delta_{11} & \Delta_{12} & \tau^{\alpha} \alpha^{-1} A^{\mathrm{T}} & F_{1}^{\mathrm{T}} & \lambda P E \\
\star & \Delta_{22} & \tau^{\alpha} \alpha^{-1}\left(A_{\tau}^{\mathrm{T}}-K^{\mathrm{T}} B^{\mathrm{T}}\right) & F_{2}^{\mathrm{T}}-K^{\mathrm{T}} F_{3}^{\mathrm{T}} & 0 \\
\star & \star & -\tau^{\alpha} \alpha^{-1} I & 0 & \lambda \tau^{\alpha} \alpha^{-1} E \\
\star & \star & \star & -\lambda I & 0 \\
\star & \star & \star & \star & -\lambda I
\end{array}\right]<0
$$

where

$$
\begin{aligned}
& \Delta_{11}=P A+A^{\mathrm{T}} P+Q_{1}+\tau^{\alpha} \alpha^{-1} I+\mu P, \\
& \Delta_{12}=P A_{\tau}-P B K, \\
& \Delta_{22}=K^{\mathrm{T}} Q_{2} K-P .
\end{aligned}
$$

By using Schur complement, $\Delta<0$ is equivalent to

$$
\left[\begin{array}{cccccccc}
\mathrm{Y}_{11} & \mathrm{Y}_{12} & \mathrm{Y}_{13} & F_{1}^{\mathrm{T}} & \lambda P E & Q_{1} & 0 & \tau^{\alpha} \alpha^{-1} I \\
\star & -P & \mathrm{Y}_{23} & F_{2}^{\mathrm{T}}-K^{\mathrm{T}} F_{3}^{\mathrm{T}} & 0 & 0 & K^{\mathrm{T}} Q_{2} & 0 \\
\star & \star & -\tau^{\alpha} \alpha^{-1} I & 0 & \mathrm{Y}_{35} & 0 & 0 & 0 \\
\star & \star & \star & -\lambda I & 0 & 0 & 0 & 0 \\
\star & \star & \star & \star & -\lambda I & 0 & 0 & 0 \\
\star & \star & \star & \star & \star & -Q_{1} & 0 & 0 \\
\star & \star & \star & \star & \star & \star & -Q_{2} & 0 \\
\star & \star & \star & \star & \star & \star & \star & -\tau^{\alpha} \alpha^{-1} I
\end{array}\right]<0,
$$

where

$$
\begin{array}{ll}
\mathrm{Y}_{11}=P A+A^{\mathrm{T}} P+\mu P, & \mathrm{Y}_{12}=P A_{\tau}-P B K \\
\mathrm{Y}_{13}=\tau^{\alpha} \alpha^{-1} A^{\mathrm{T}}, & \mathrm{Y}_{23}=\tau^{\alpha} \alpha^{-1}\left(A_{\tau}^{\mathrm{T}}-K^{\mathrm{T}} B^{\mathrm{T}}\right), \\
\mathrm{Y}_{24}=F_{2}^{\mathrm{T}}-K^{\mathrm{T}} F_{3}^{\mathrm{T}}, & \mathrm{Y}_{35}=\lambda \tau^{\alpha} \alpha^{-1} E .
\end{array}
$$

By pre- and post-multiplying (15) by $\operatorname{diag}\left\{P^{-1}, P^{-1}, I, I, I, I, I, I\right\}$ yields

$$
\left[\begin{array}{cccccccc}
\Gamma_{11} & \Gamma_{12} & \Gamma_{13} & P^{-1} F_{1}^{\mathrm{T}} & \lambda E & P^{-1} Q_{1} & 0 & \tau^{\alpha} \alpha^{-1} P^{-1} \\
\star & -P^{-1} & \Gamma_{23} & \Gamma_{24} & 0 & 0 & Y^{\mathrm{T}} Q_{2} & 0 \\
\star & \star & -\tau^{\alpha} \alpha^{-1} I & 0 & \lambda \tau^{\alpha} \alpha^{-1} E & 0 & 0 & 0 \\
\star & \star & \star & -\lambda I & 0 & 0 & 0 & 0 \\
\star & \star & \star & \star & -\lambda I & 0 & 0 & 0 \\
\star & \star & \star & \star & \star & -Q_{1} & 0 & 0 \\
\star & \star & \star & \star & \star & \star & -Q_{2} & 0 \\
\star & \star & \star & \star & \star & \star & \star & -\tau^{\alpha} \alpha^{-1} I
\end{array}\right]<0,
$$

where

$$
\begin{array}{ll}
\Gamma_{11}=A P^{-1}+P^{-1} A^{\mathrm{T}}+\mu P^{-1}, & \Gamma_{12}=A_{\tau} P^{-1}-B K P^{-1} \\
\Gamma_{13}=\tau^{\alpha} \alpha^{-1} P^{-1} A^{\mathrm{T}}, & \Gamma_{23}=\tau^{\alpha} \alpha^{-1}\left(P^{-1} A_{\tau}^{\mathrm{T}}-P^{-1} K^{\mathrm{T}} B^{\mathrm{T}}\right), \\
\Gamma_{24}=P^{-1} F_{2}^{\mathrm{T}}-P^{-1} K^{\mathrm{T}} F_{3}^{\mathrm{T}} . &
\end{array}
$$

If we take $\mu \rightarrow 1^{+}$, and have $P^{-1}=\bar{P}, K P^{-1}=K \bar{P}=Y$ in (16), then, expression (16) can be described as (6). Therefore, by using Lemma 3, the closed-loop control system (4) under the controller (3) is asymptotically stable,

$$
D^{\alpha} V(x(t)) \leq-x^{\mathrm{T}}(t) Q_{1} x(t)-x^{\mathrm{T}}(t) K^{\mathrm{T}} Q_{2} K x(t)<0 .
$$


Integrating with order $\alpha$ both sides of (17) from 0 to $T_{1}$, and taking Lemma 1 into consideration, one has

$$
V\left(x\left(T_{1}\right)\right)-V(x(0)) \leq-J(u) .
$$

Since $V\left(x\left(T_{1}\right)\right) \geq 0$, one has

$$
J(u) \leq V(x(0))-V\left(x\left(T_{1}\right)\right) \leq V(x(0)) \leq \lambda_{\max }\left(\bar{P}^{-1}\right)\|\phi\|^{2}=J^{*} .
$$

This completes the proof of the theorem.

Remark 2. If $\alpha=1$ in the above quadratic cost function (2), then it will degenerated into the definition of cost function in integer-order systems which were discussed in the literature [22-31].

Remark 3. In the control of real-plant including FO delayed systems, it is far from enough to design a controller which not only robustly stabilises the uncertain system, but also ensures that an sufficient performance level needs to be guaranteed. One effective way to solve this problem is the so-called guaranteed cost control method. Several papers [37-41] discussed the control of FO delayed systems, but the performance level was not taken into account.

Remark 4. The problem of finite-time and robust guaranteed cost control of uncertain fractionalorder neural networks were investigated in [42,43], and the output-feedback-guaranteed cost control of a class of FO uncertain linear systems with time delay was discussed in [44]. However, these results are delay-and order-independent. Here, delay-and order-dependent controller methods are obtained, which are less conservative than those presented in [42-44].

Remark 5. Chen et al. [44] studied the guaranteed cost controller design for a class of FO uncertain delayed linear systems by using the output-feedback controller without delay. Here, time delays both in the state and the input are discussed.

Remark 6. The upper bound of the cost function is given herein, but it is not optimal. In fact, the guaranteed cost in (2) depends on the choice of guaranteed cost controllers. The algorithm that minimizes the cost in (2) is of interest and such a controller is said to be an optimal guaranteed cost controller. Thus, the design problem of the optimal guaranteed cost controller can be formulated as an optimization problem. For more details on this topic, readers can refer to [45-47]. Nonetheless, it remains for future work the design of the optimal guaranteed cost controller.

\section{Numerical Examples}

In this section, two examples will be given to show the effectiveness of the proposed theoretical results with computer simulations. The numerical method proposed in [48] is used in the follow-up.

Example 1. Consider the following uncertain FO linear system (1) with parameters

$$
\begin{aligned}
& A=\left[\begin{array}{cc}
-2 & 3 \\
-1 & -2
\end{array}\right], A_{\tau}=\left[\begin{array}{ll}
1 & 1 \\
2 & 0
\end{array}\right], B=\left[\begin{array}{c}
1 \\
-2
\end{array}\right], \\
& E_{1}=\left[\begin{array}{l}
0.1 \\
0.1
\end{array}\right], F_{1}=\left[\begin{array}{ll}
0.1 & 0.1
\end{array}\right], F_{2}=\left[\begin{array}{ll}
-0.1 & 0.2
\end{array}\right], \\
& F_{3}=-0.1, \alpha=0.9, \tau=0.1 .
\end{aligned}
$$

Suppose that all states of the system are measurable. Using the MATLAB LMI toolbox, one obtains the feasible solution as follows from Theorem 1

$$
\bar{P}=\left[\begin{array}{cc}
0.1161 & -0.0367 \\
-0.0367 & 0.0392
\end{array}\right], Y=\left[\begin{array}{ll}
0.1374 & -0.0411
\end{array}\right], \lambda=0.9689 .
$$


The controller gain matrix is given by

$$
K=Y \tilde{P}^{-1}=\left[\begin{array}{ll}
1.2115 & 0.0876
\end{array}\right] .
$$

Hence, the above results show that all the conditions stated in Theorem 1 have been satisfied and that the system can be stabilized by the full state controller.

Let us consider $h(t)=\cos t$ and the initial state $\phi=\left[\begin{array}{ll}2 & -3\end{array}\right]^{T}$. Figures 1 and 2 show the minimal upper bound of the generalized cost $J^{*}=530.6873$, time response of the selected system without and with the control input, respectively. Form the two figures, we can observe that the states of system without input control is divergent, and that the controlled system is convergent.

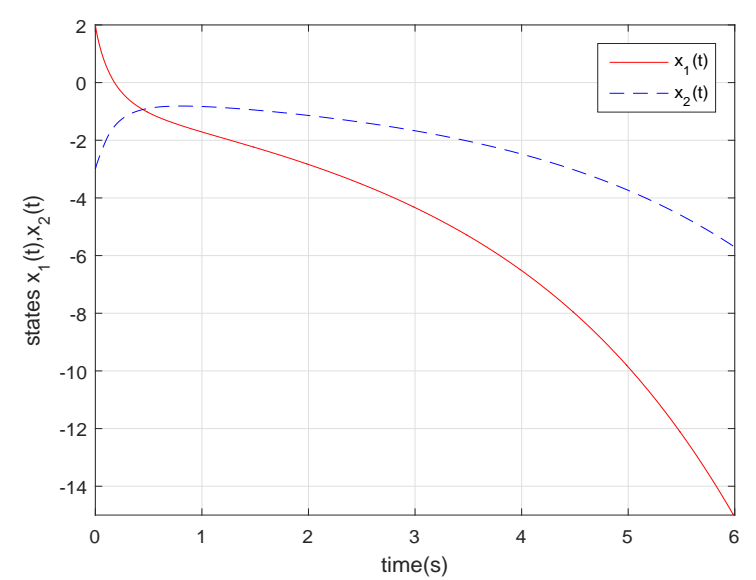

Figure 1. Time response of the selected systems without control input in the Example 1.

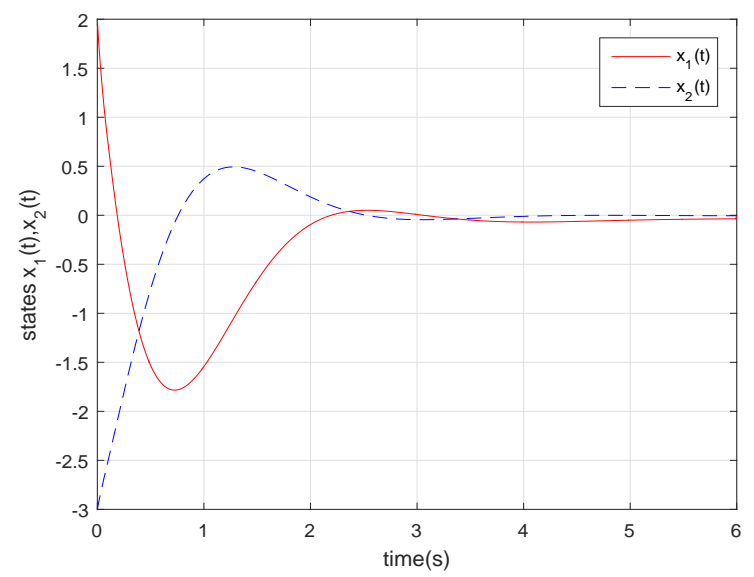

Figure 2. Time response of the selected systems with control input in the Example 1.

Example 2. Consider the following uncertain FO linear system (1) with parameters

$$
\begin{aligned}
A & =\left[\begin{array}{ccc}
-3 & 2 & 1 \\
2 & -5 & 1 \\
3 & 1 & -4
\end{array}\right], A_{\tau}=\left[\begin{array}{ccc}
1 & 0 & 0 \\
0 & 1 & 0 \\
-1 & 0 & -1
\end{array}\right], B=\left[\begin{array}{l}
1 \\
1 \\
0
\end{array}\right], \\
E_{1} & =\left[\begin{array}{l}
0.1 \\
0.1 \\
0.2
\end{array}\right], F_{1}=\left[\begin{array}{lll}
0.1 & 0.1 & 0.1
\end{array}\right], F_{2}=\left[\begin{array}{lll}
0.1 & 0.1 & 0.1
\end{array}\right], \\
F_{3} & =0.1, \alpha=0.9, \tau=0.1 .
\end{aligned}
$$


By using the MATLAB LMI toolbox, one obtains the feasible solution as follows from Theorem 1

$$
\begin{aligned}
\bar{P}= & {\left[\begin{array}{ccc}
0.2350 & -0.2146 & -0.2245 \\
-0.2146 & 0.7076 & -0.3655 \\
-0.2245 & -0.3655 & 0.9720
\end{array}\right], Y=\left[\begin{array}{ccc}
-0.0657 & -0.2903 & 0.4868
\end{array}\right], } \\
& \lambda=0.9689 .
\end{aligned}
$$

The controller gain matrix is as follows:

$$
K=Y \tilde{P}^{-1}=\left[\begin{array}{lll}
-0.3598 & -0.3767 & 0.2760
\end{array}\right] .
$$

Hence, the above results show that all the conditions stated in Theorem 1 have been satisfied and that the system can be stabilized by the full state controller.

Consider $h(t)=\sin t$ and the initial state $\phi=\left[\begin{array}{lll}1 & -2 & 3\end{array}\right]^{T}$. Figures 3 and 4 show the minimal upper bound of the generalized cost $J^{*}=1234.3$, time response of the selected system without and with the control input, respectively.

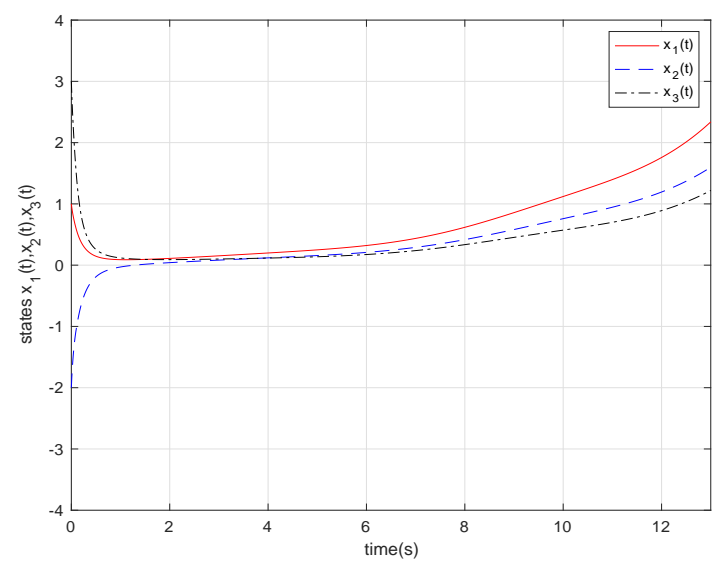

Figure 3. Time response of the selected systems without control input in the Example 2.

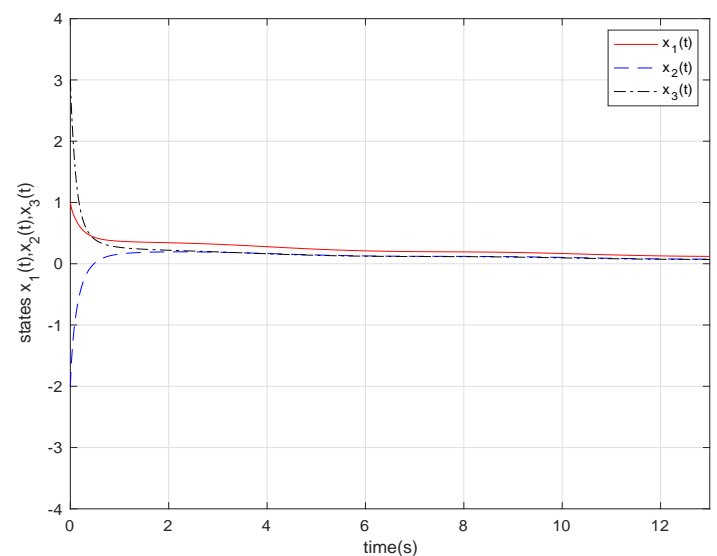

Figure 4. Time response of the selected systems with control input in the Example 2.

\section{Conclusions}

This paper discussed the problem of guaranteed cost control for uncertain FO linear systems with time delays both in the state and the input by employing the FO Razumikhin theorem and a new definition of guaranteed cost function for FO systems. Delay- and order-dependent conditions were developed, and state-feedback controllers designed. The new approach guarantees the robust stability of the closed-loop system and an upper 
bound of the specified linear integral-quadratic cost function for all delays. Illustrative examples demonstrated the applicability and effectiveness of the proposed method.

Author Contributions: F.Q., simulation, writing and editing the manuscript; Y.C., supervision, and project administration; L.C., methodology formal analysis; J.A.T.M., writing-review and editing. All authors have read and agreed to the published version of the manuscript.

Funding: This work was supported by the National Natural Science Funds of China (Nos. 62073114; 61633005).

Institutional Review Board Statement: Not applicable.

Informed Consent Statement: Not applicable.

Data Availability Statement: No new data were created or analyzed in this study. Data sharing is not applicable to this article.

Conflicts of Interest: The authors declare no conflict of interest.

\section{References}

1. Tepljakov, A. Fractional-Order Modeling and Control of Dynamic Systems; Springer: Berlin, Germany, 2017.

2. Yang, X. General Fractional Derivatives: Theory, Methods and Applications; CRC Press: Boca Raton, FL, USA, 2019.

3. Daftardar-Gejji, V. Fractional Calculus and Fractional Differential Equations; Springer: Berlin, Germany, 2019.

4. Hilfer, R. Applications of Fractional Calculus in Physics; World Scientific: Singapore, 2000.

5. Laskin, N. Fractional Quantum Mechanics; World Scientific: Singapore, 2018.

6. West, B. Fractional Calculus View of Complexity: Tomorrows Science; CRC Press: Boca Raton, FL, USA, 2016.

7. Singh, H.; Kumar, D.; Baleanu, D. Methods of Mathematical Modelling: Fractional Differential Equations; CRC Press: Boca Raton, FL, USA, 2019.

8. Luo, Y.; Chen, Y. Fractional Order Motion Controls; Wiley: Hoboken, NJ, USA, 2012.

9. Ostalczyk, P. Discrete Fractional Calculus: Applications in Control and Image Processing; World Scientific: Singapore, $2015 ;$ Volume 4.

10. Chen, L.; Li, T.; Chen, Y.; Wu, R.; Ge, S. Robust passivity and feedback passification of a class of uncertain fractional-order linear systems. Int. J. Syst. Sci. 2019, 50, 1149-1162. [CrossRef]

11. Xue, D. Fractional-Order Control Systems: Fundamentals and Numerical Implementations; De Gruyter: Berlin, Germany, 2017.

12. Ge, F.; Chen, Y.; Kou, C. Regional Analysis of Time-Fractional Diffusion Processes; Springer: Berlin, Germany, 2018.

13. Lan, Y.H.; Huang, H.X.; Zhou, Y. Observer-based robust control of $a(1 \leq a<2)$ fractional-order uncertain systems: A linear matrix inequality approach. IET Control Theory Appl. 2012, 6, 229-234.

14. Badri, P.; Sojoodi, M. Stability and stabilization of fractional-order systems with different derivative orders: An LMI approach. Asian J. Control 2019, 21, 2270-2279. [CrossRef]

15. Chen, L.; Wu, R.; He, Y.; Yin, L. Robust stability and stabilization of fractional-order linear systems with polytopic uncertainties. Appl. Math. Comput. 2015, 257, 274-284. [CrossRef]

16. Shahri, E.S.A.; Alfi, A.; Machado, J.T. Lyapunov method for the stability analysis of uncertain fractional-order systems under input saturation. Appl. Math. Model. 2020, 81, 663-672. [CrossRef]

17. Mohsenipour, R.; Liu, X. Robust D-stability test of LTI general fractional order control systems. IEEE/CAA J. Autom. Sin. 2020, 7, 853-864. [CrossRef]

18. N'Doye, I.; Darouach, M.; Zasadzinski, M.; Radhy, N.E. Robust stabilization of uncertain descriptor fractional-order systems. Automatica 2013, 49, 1907-1913. [CrossRef]

19. Ma, Y.; Lu, J.; Chen, W. Robust stability and stabilization of fractional order linear systems with positive real uncertainty. ISA Trans. 2014, 53, 199-209. [CrossRef]

20. Dinh, C.H.; Mai, V.T.; Duong, T.H. New Results on Stability and Stabilization of Delayed Caputo Fractional Order Systems with Convex Polytopic Uncertainties. J. Syst. Sci. Complex. 2020, 33, 563-583. [CrossRef]

21. Chang, S.; Peng, T. Adaptive guaranteed cost control of systems with uncertain parameters. IEEE Trans. Autom. Control 1972, 17, 474-483. [CrossRef]

22. Chang, X.; Huang, R.; Park, J. Robust guaranteed cost control under digital communication channels. IEEE Trans. Ind. Inf. 2019, 16, 319-327. [CrossRef]

23. Zong, G.; Ren, H. Guaranteed cost finite-time control for semi-Markov jump systems with event-triggered scheme and quantization input. Int. J. Robust Nonlinear Control 2019, 29, 5251-5273. [CrossRef]

24. Li, Y. Optimal guaranteed cost control of linear uncertain system: an LMI approach. Control Theory Appl. 2000, 17, 423-428.

25. Xu, H.; Teo, K.L.; Liu, X. Robust stability analysis of guaranteed cost control for impulsive switched systems. IEEE Trans. Syst. Man Cybern. Part B 2008, 38, 1419-1422.

26. Li, H.; Wang, J.; Wu, L.; Lam, H.; Gao, Y. Optimal guaranteed cost sliding-mode control of interval type-2 fuzzy time-delay systems. IEEE Trans. Fuzzy Syst. 2018, 26, 246-257. [CrossRef] 
27. Xiang, C.; Petersen, I.R.; Dong, D. Performance analysis and coherent guaranteed cost control for uncertain quantum systems using small gain and Popov methods. IEEE Trans. Autom. Control 2016, 62, 1524-1529. [CrossRef]

28. Yan, Z.; Park, J.H.; Zhang, W. Finite-time guaranteed cost control for Itô Stochastic Markovian jump systems with incomplete transition rates. Int. J. Robust Nonlinear Control 2017, 27, 66-83. [CrossRef]

29. Gyurkovics, E. Guaranteed cost control of discrete-time uncertain systems with both state and input delays. Int. J. Control 2016, 89, 2073-2082. [CrossRef]

30. Chen, W.; Xu, J.; Guan, Z. Guaranteed cost control for uncertain Markovian jump systems with mode-dependent time-delays. IEEE Trans. Autom. Control 2003, 48, 2270-2277. [CrossRef]

31. Xu, S.; Lam, J.; Yang, C.; Verriest, E. An LMI approach to guaranteed cost control for uncertain linear neutral delay systems. Int. J. Robust Nonlinear Control 2003, 13, 35-53. [CrossRef]

32. Podlubny, I. Fractional Differential Equations; Academic Press: San Diego, CA, USA, 1999.

33. Liang, S.; Wu, R.; Chen, L. Adaptive pinning synchronization in fractional-order uncertain complex dynamical networks with delay. Physica A 2016, 444, 49-62. [CrossRef]

34. Chen, B.; Chen, J. Razumikhin-type stability theorems for functional fractional-order differential systems and applications. Appl. Math. Comput. 2015, 254, 63-69. [CrossRef]

35. Xie, L. Output feedback $H_{\infty}$ control of systems with parameter uncertainty. Int. J. Control 1996, 63, 741-750. [CrossRef]

36. Last, E. Linear Matrix Inequalities in System and Control Theory. Proc. IEEE 1994, 86, 2473-2474.

37. Si-Ammour, A.; Djennoune, S.; Bettayeb, M. A sliding mode control for linear fractional systems with input and state delays. Commun. Nonlinear Sci. Numerical Simul. 2009, 14, 2310-2318. [CrossRef]

38. Li, Y.; Jiang, W. Fractional order nonlinear systems with delay in iterative learning control. Appl. Math. Comput. 2015, 257, 546-552.

39. Geng, W.; Lin, C.; Chen, B. Observer-based stabilizing control for fractional-order systems with input delay. ISA Trans. 2020, 100, 103-108. [CrossRef]

40. Choudhary, N.; Sivaramakrishnan, J.; Kar, I.N. Sliding mode control of uncertain fractional order systems with delay. Int. J. Control 2020, 93, 934-943. [CrossRef]

41. Liu, H.; Xie, G.; Gao, Y. Containment control of fractional-order multi-agent systems with time-varying delays. J. Frankl. Inst. 2019, 356, 9992-10014. [CrossRef]

42. Thuan, M.V.; Binh, T.N.; Huong, D.C. Finite-time guaranteed cost control of Caputo fractional-order neural networks. Asian J. Control 2020, 22, 696-705. [CrossRef]

43. Thuan, M.V.; Huong, D.C. Robust guaranteed cost control for time-delay fractional-order neural networks systems. Optim. Control Appl. Methods 2019, 40, 613-625. [CrossRef]

44. Chen, L.; Li, T.; Wu, R.; Lopes, A.M.; Machado, J.T.; Wu, K. Output-feedback-guaranteed cost control of fractional-order uncertain linear delayed systems. Comput. Appl. Math. 2020, 39, 1-18. [CrossRef]

45. Church, K.E.; Liu, X. Cost-Effective Robust Stabilization and Bifurcation Suppression. SIAM J. Control Optim. 2019, 57, 2240-2268. [CrossRef]

46. Xie, X.; Lam, J. Guaranteed cost control of periodic piecewise linear time-delay systems. Automatica 2018, 94, 274-282. [CrossRef]

47. Rahimkhani, P.; Ordokhani, Y.; Babolian, E. An efficient approximate method for solving delay fractional optimal control problems. Nonlinear Dyn. 2016, 86, 1649-1661. [CrossRef]

48. Wang, H.; Gu, Y.; Yu, Y. Numerical solution of fractional-order time-varying delayed differential systems using Lagrange interpolation. Nonlinear Dyn. 2019, 95, 809-822. [CrossRef] 